\title{
NOUVELLES DONNÉES SUR L'INSÉMINATION ARTIFICIELLE PORCINE RÉSULTATS PRATIQUES
}

PAR

\section{F. DU MESNIL DU BUISSON et L. DAUZIER ( $\left.{ }^{1}\right)$}

Station de Recherches de Physiologie animale, Centre national de Recherches zootechniques, Jouy-en-Josas.

I'importance de la production porcine en France est telle que toute amélioration de la qualité du cheptel est susceptible d'entraîner des économies considérables sur le plan national.

Cinquante ans de sélection poursuivie par les organisations d'élevage des grandes races ont permis d'améliorer considérablement la conformation des géniteurs ; et l'appréciation des Verrats sur la qualité de leur descendance, entreprise depuis peu, doit permettre de déceler, parmi les meilleures lignées, celles dont l'indice de consommation est le plus faible. Ces efforts et ces réalisations ne pourront porter leurs fruits sur le plan national que par une utilisation maximum des géniteurs d'élite. L'insémination artificielle doit nous en fournir la possibilité.

En plus de cet avantage proprement zootechnique, l'insémination porcine représente un excellent moyen de lutte contre les épizooties répandues par les déplacements d'animaux et leur contact au moment de 1'accouplement.

Cependant la raison principale qui pousse actuellement la masse des éleveurs à s'intéresser à l'insémination artificielle porcine tient aux tracas que cause la saillie d'une truie avec un verrat presque toujours éloigné et, de surcroît, souvent médiocre.

Toutes ces raisons expliquent pourquoi depuis quelques années, devant le succès de l'insémination artificielle bovine, un certain nombre de chercheurs ont tenté d'appliquer cette technique à l'élevage porcin.

Le travail des Japonais ITo, Nrwa et Kudo, à partir de I947, est le premier qui porte sur plusieurs centaines d'animaux; une expérience a été rapportée ensuite aux Philippines par CoroNEL en I953. En France,

(1) Avec la collaboration technique de J. Goussopoulos, F. Renard, J. P. Vincent et Eliane Callanguin.

Nos remerciements aux responsables des Centres d'Insémination artificielle de Charmoy et de Poitiers dont la collaboration, l'hospitalité et l'aide matérielle nous ont été précieuses au cours de ces essais. 
à partir de 1953 également, des recherches ont été entreprises par MAULEON et nous-mêmes, et peu après, en Angleterre, par POLGE et dernièrement par Aamdal, en Norvège.

Outre les données acquises par près de 20 ans d'insémination artificielle bovine, les chercheurs ont pu utiliser un certain nombre de travaux sur la physiologie de la reproduction du Porc dont les plus importants ont eu pour objet le sperme et sa production (Mc KENZIE, MILLER et BAUGuess, I938; ManN, depuis I95I), les organes génitaux et le cycle sexuel de la Truie (Corner, I9I5, I92 I ; Burger, I952), 1'accouplement (RODOLFO, I934), 1'endocrinologie femelle expérimentale (CASIDA et collaborateurs, I952), et l'insémination artificielle elle-même, celle-ci ayant été réalisée dès I932 sur quelques truies, par Milovanov.

Or, malgré les renseignements ainsi obtenus, malgré des résultats convenables enregistrés en laboratoire et malgré des conditions économiques favorables, l'insémination artificielle du Porc n'a pris d'essor en aucun pays jusqu'à ce jour car les résultats dans la pratique se sont jusqu'ici toujours montrés assez décevants.

Cependant, les essais pratiques restent les set1s qui soient susceptibles de poser les problèmes dans les conditions où un centre d'insémination les rencontrera.

C'est pourquoi nous publions ici les résultats de 3 essais d'insémination réalisés dans les fermes en I955 et I956, et les conclusions qu'on peut en tirer aussi bien pour sa mise en pratique que pour la solution des problèmes particuliers de physiologie sexuelle que pose le Pore.

\section{I. - MATÉRIEL ET MÉTHODES DE COLLECTE. CONSERVATION DU SPERME}

La méthode de collecte comme les instruments ont été étudiés et mis au point depuis longtemps.

Que le vagin artificiel soit court (ITO, NIWA et KUDO, I948 a) ou long (MC KENZIE, I93I ; RODOLFO, I934 $a$ ), avec ou sans variation possible de pression, il semble que le Verrat se laisse collecter assez facilement. Pour notre part, nous avons toujours utilisé un vagin de $30 \mathrm{~cm}$ de long muni d'une poire qui permet d'obtenir une pression variable selon la méthode de Milovanov (I934).

L'usage d'un mannequin à hauteur réglable est extrêmement commode ; il est dérivé du modèle réalisé par RoDoLFo en I934. Le mannequin dont nous nous servons est couvert de cuir et la présence d'une vis sans fin sous chacun de ses pieds permet à la fois un réglage en hauteur et surtout une bonne stabilité sur un terrain inégal.

De nombreux auteurs ont décrit après RoDOL,Fo les différentes phases de l'éjaculation. Notre expérience personnelle porte sur des verrats de race Large White. 
I a première phase correspond à l'élimination de la partie préspermatique. Des sécrétions accessoires peu abondantes s'écoulent en même temps que le pénis du verrat effectue des mouvements d'intromission et de retrait, le verrat lui-même étant souvent agité.

La deuxième phase, de courte durée ( 2 à 3 minutes) correspond à un calme absolu du Verrat. Le sperme, laiteux, coule abondamment. Cette fraction est très riche en spermatozoïdes : dans le quart du volume émis $(60$ à $80 \mathrm{cc}$ ) on retrouve les $2 / 3$ des spermatozoïdes de l'éjaculat total $(250 \mathrm{cc})$.

I a troisième phase commence souvent par une période d'agitation sans éjaculation. Ensuite coule une sécrétion abondante (200 cc environ), assez claire; la concentration en spermatozoïdes y est très faible (de l'ordre de Ioo ooo par $\mathrm{mm}^{3}$ ).

TABLEAU I

\begin{tabular}{|c|c|c|c|}
\hline \multirow{2}{*}{ Jintervalle entre les collectes } & \multirow{2}{*}{$\begin{array}{l}\text { Nombre des } \\
\text { Collectes }\end{array}$} & \multicolumn{2}{|c|}{$\begin{array}{c}\text { Nombre de spermatozoïd s } \\
\text { par collecte }\left(\mathrm{IO}^{\theta}\right)\end{array}$} \\
\hline & & Moyenne & Ecart \\
\hline 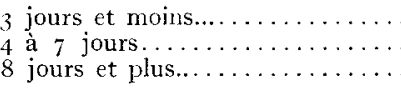 & $\begin{array}{r}49 \\
110 \\
91\end{array}$ & $\begin{array}{l}51,5 \\
69,8 \\
90,7\end{array}$ & $\begin{array}{l}\mathrm{II}, 4-84,5 \\
33,2-\mathrm{I}_{1,2} \\
49,5-\mathrm{I} 64,8\end{array}$ \\
\hline
\end{tabular}

Vers la fin de cette phase, le pénis se retire doucement en même temps que l'éjaculation se termine par des sécrétions qui prennent en masse rapidement formant un bouchon que l'on trouve dans le vagin artificiel. L'absence de ce bouchon dénote une collecte incomplète.

En plus de la masse gélatineuse qui forme ce bouchon, on trouve, dans le sperme même, des grains blanchâtres ressemblant à du tapioca cuit et qui gonflent au contact du sperme ou de l'eau : ils sont émis durant les 3 phases mais principalement pendant la dernière.

Le volume de sperme émis est assez constant, le chiffre moyen étant de $250 \mathrm{~cm}^{3}$ y compris la partie solide ; nous avons obtenu aul maximum $596 \mathrm{cc}$ pour un même éjaculat. Au contraire, le nombre des spermatozoïdes par collecte angmente lorsque l'on espace les récoltes : de 50 milliards environ quand les collectes sont effectuées à trois jours d'intervalle ou moins, on passe à plus de 80 milliards avec une collecte hebdomadaire et on dépasse fréquemment roo milliards avec des collectes plus espacées encore. Le maximum de spermatozoïdes que nous ayons obtenu a été de 20 I milliards pour une même collecte. Le tableau I indique les chiffres moyens calculés à partir de 250 collectes.

Outre la diminution du nombre de spermatozoïdes par éjaculat det1x autres raisons nous poussent à recommander un espacement suffisant des collectes dans la pratique de l'insémination artificielle. 
D'une part le sperme perd une partie de son aptitude à la survie lorsque l'intervalle entre les collectes est plus court que $7^{2}$ heures, fait constaté par Mc Kenzie, MiLIER et BAUGuess (I938), et confirmé par ITo, Niwa, Kudo et Mizuho (I948). D'autre part, bien qu'il soit démontré qu'un Verrat est capable d'effectuer spontanément un grand nombre de saillies, pendant une courte période (II fois en 37 heures, BURGER, I952), il est facile de constater que des collectes effectuées tous les deux jours par exemple, amènent assez rapidement le Verrat à un dégoût du mannequin. Ainsi, une collecte tous les 4 à 7 jours représente, à notre avis, la fréquence la plus satisfaisante.

Nous avons abordé ailleurs l'étude de la conservation du sperme de Verrat dilué ou non (Du MEsniL DU Buisson, r956-r957) ; cependant, rappelons deux caractéristiques du sperme qui doivent retenir notre attention, comme particulièrement importantes pour la pratique de l'insémination artificielle :

- C'est d'abord l'état d'anabiose (Mrlovanov et Habibulin, I933), dans lequel tombe le sperme pur, conservé, et qui peut faire croire à sa mort, alors qu'après agitation à l'air et réchauffement à plus de $31^{\circ}$, on constate qu'il peut retrouver sa vitalité initiale.

- C'est ensuite l'amélioration très nette de la conservation que l'on obtient en diluant le sperme dans des milieux salins additionnés de jaune d'œuf ou de lait. Dans ces conditions, la température de conservation optimum se situe autour de $7^{\circ}$, alors que, pour la conservation du sperme non dilué, la température ambiante semble préférable. Rappelons également l'effet bénéfique qu'apporte le bicarbonate de sodium au sperme dilué, de même que le gaz carbonique au sperme conservé pur.

Bien que nous ayons obtenu (DU MESNIL DU Buisson, I956) des fécondations avec du sperme conservé 48 heures dans du lait, nous n'avons jamais utilisé dans la pratique, du sperme conservé plus de 8 heures. En attendant son utilisation, le sperme demeurait à la température ambiante $\left(\mathrm{I} 5\right.$ à $\left.20^{\circ} \mathrm{C}\right)$. En général, il a été dilué immédiatement après la collecte. Dans un lot, cependant, nous l'avons conservé pur, la dilution n'ayant eu lieu qu'extemporanément.

\section{II. - TECHNIQUE D'INSÉMINATION ARTIFICIELLE PROPREMENT DITE}

I a rencontre des spermatozoïdes et des œufs dans les meilleurs délais étant la condition nécessaire au succès de l'insémination, le choix du moment de l'insémination et, compte tenu de la physiologie particulière de l'utérus de la Truie (Du MEsnil Du Buisson et DauzIER, I955 a et $b$ ), celui du mode d'insémination sont extrêmement importants dans la pratique. 


\section{Période favorable pour l'insémination}

\section{a) Détection des chaleurs.}

La durée des chaleurs de la Truie ( 2 à 4 jours en général) la place dans une position intermédiaire entre la Vache et la Jument; variable avec les races, cette durée l'est aussi avec l'âge (BURGER, I952), elle dépasse rarement deux jours et demi chez les nullipares. Le comportement de la Truie est un bon critère, mais il ne peut être apprécié que par comparaison avec l'attitude habituelle de la bête. La plupart du temps le rut est caractérisé par une grande placidité de l'animal. La Truie "s'arrête", elle refuse d'avancer si on la pousse vers l'avant; en lui frottant le dos on provoque une certaine tension musculaire de tout le corps et un redressement des oreilles, qui peuvent faire penser à la lordose-réflexe obtenue chez la Ratte en œstrus.

TABLEAU II

\begin{tabular}{|c|c|c|c|}
\hline Résultat de l'examen vaginal & $\begin{array}{c}\text { Nombre de Truies } \\
\text { examinées }\end{array}$ & $\begin{array}{l}\text { Nombre de Truies } \\
\text { pleines, après } \\
\text { insémination }\end{array}$ & Pourcentage \\
\hline 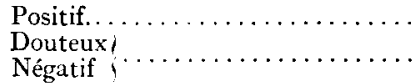 & $\begin{array}{l}39 \\
3^{2}\end{array}$ & $\begin{array}{l}\text { I9 } \\
\text { II }\end{array}$ & $\begin{array}{l}48,7 \% \\
34,3 \%\end{array}$ \\
\hline Totaux..... & $7 \mathrm{I}$ & 30 & $42,2 \%$ \\
\hline
\end{tabular}

Malheureusement, la généralisation paraît impossible, certaines truies ayant au contraire un comportement très agité, grognant, refusant toute nourriture.

L orsque plusieurs truies sont élevées ensemble, et que 1'une est en chaleurs, il est fréquent qu'elle chevauche les autres ou qu'elle soit chevauchée par elles, mais là encore, la règle n'est pas générale et quelquefois, on remarque ce comportement également avant que l'œstrus ne soit réellement atteint. De même, le gonflement de la vulve qui accompagne généralement l'œstrus, le précède quelquefois de plusieurs jours, surtout pour les premières chaleurs.

Les manifestations vulvaires sont accompagnées de modifications cytologiques (WiLson, rg26) qui, elles aussi, ont lieu de façon trop progressive pour pouvoir être utilisées comme test pour la détection de l'œstrus.

En appliquant sur $7 \mathrm{I}$ truies inséminées, les méthodes de colorations classiques pour les frottis vaginaux (CASTOR, I950), nous n'avons obtenu aucune indication permettant une meilleure précision.

On voit sur le tableau II que les pourcentages de gestation restent 
encore relativement élevés chez les truies dont l'examen vaginal a fourni un résultat douteux ou négatif, quant à l'existence de 1'œstrus. La méthode de Thiery (I952) ne nous a pas fourni de données plus précises. Ces résultats rejoignent les constatations de STEINBaCh (I95I).

\section{b) Le moment de l'ovulation.}

Les avis sont très partagés sur le moment de l'ovulation et BURGER (I952) cite I2 auteurs dont les avis diffèrent quant au moment opportun pour l'accouplement par rapport au début des chaleurs. Bien que l'on sache depuis CORNER (I9I5) que l'ovulation intervient dans tous les cas, il semble que le nombre d'ovocytes pondus à un instant donné de l'œstrus, soit dans certaines conditions (LEBEDEV et PITKJANEN, I95I) sous la dépendance de stimulations dues à la saillie.

Nous avons montré (DU Mesnil, DU Buisson et DaUzier, I955 b) que les spermatozoïdes demeurent vivants au moins 48 heures à la jonction utéro-tubaire; si leur pouvoir fécondant était également conservé, la détermination du moment de 1'ovulation perdrait une grande partie de son importance. En tout cas, l'ovulation se produit en général dans la $2^{\mathrm{e}}$ moitié de l'œstrus et le moment à recommander pour l'insémination semble être le milieu de l'œstrus.

\section{2o Le mode d'insémination}

If'insémination naturelle a été étudiée par RoDOLFo en I934, ensuite par divers auteurs : Kvasnirski (I95I), DU MESNiL DU Buisson et Dauzier (1955), Mann, Polge et Rowson (I956). Elle se fait directement in utero. Une résorption très rapide de tous les éléments du sperme dans 1'utérus, y compris les spermatozoïdes se produit en moins de 5 heures après la saillie. Les seuls spermatozoïdes qui puissent concourir à la fécondation sont ceux qui atteignent la jonction utéro-tubaire avant que la lyse observée dans l'utérus n'intervienne. Pour la pratique de 1'insémination, nous pensons donc que l'essentiel consiste à permettre aux spermatozoïdes d'atteindre dans les délais les plus brefs, la jonction utéro-tubaire où ils trouvent un refuge en attendant leur utilisation pour la fécondation.

Pour faire pénétrer le dilueur dans l'utérus, divers instruments spéciaux ont été proposés car les tubérosités du col montrent durant 1'œstrus, un état de turgescence et de tonicité qui interdit de le traverser avec un catheter rigide. Pour que le sperme passe dans l'utérus, sans refluer dans le vagin, il faut que l'extrémité de la sonde s'accole aussi intimement que possible à la lumière sinueuse du col. Les Japonais utilisent pour cela un petit tube de I à $2 \mathrm{~cm}$ fixé obliquement, par rapport 
à l'axe d'un corps de pompe qui pénètre lui-même profondément dans le vagin.

Pour notre part, après avoir utilisé une sonde œsophagienne munie d'un ballonnet gonflable (MAULEON, I953), nous lui avons préféré la sonde plus grosse de MC KeNZIE, Milier et BAUGuess (diamètre $1,6 \mathrm{~cm}$, longueur $35 \mathrm{~cm}$ ). Le sperme est contenu soit dans une grosse seringue de Ioo cc, soit dans un récipient muni d'un bouchon à vis et d'un dispositif à poire semblable à celui qu'utilise PoLGE (I956). A la fin de chaque insémination un petit morceau de coton est placé à l'entrée du vagin.

\section{III. - LES RÉSULTATS DE L'DNSÉMINATION ARTIFICIELLE. TAUX DE GESTATION MOYEN ET CYCLES OESTRIENS ANORMAUX}

\section{Matériel et méthode}

Les chiffres que nous présentons ici groupent les résultats de 3 essais d'insémination de I mois chacun, effectués en juillet I955 et en avril et juin 1956 chez des éleveurs de 1'Yonne et de la Vienne. I.es truies inséminées, issues généralement de croisements entre Large White et races locales, étaient souvent soumises à des conditions d'élevage et d'alimentation inadéquates. 4 à 5 Verrats I arge White ont été employés durant chacune des périodes, ce nombre permettant d'effectuer sur chaque Verrat des collectes espacées de 3 à 6 jours.

La collecte effectuée chaque jour entre II h et $\mathrm{I} 4 \mathrm{~h}$ a toujours été utilisée dans l'après-midi. Après élimination des sécrétions solides par filtration sur de la gaze, l'éjaculat non fractionné fut mélangé au dilueur, lui-même préalablement porté à $3 \mathrm{I}^{\circ} \mathrm{C}$ (voir composition des dilueurs au tableau VII).

Toutes les inséminations ont été faites avec $250 \mathrm{cc}$ de sperme dilué contenant entre 2 et $\mathrm{I} 2$ milliards de spermatozoïdes : ce volume très fort de diluat a été utilisé systématiquement à la suite de nos observations (Du Mesnil DU Buisson et Dauzier, I955c) relatives à l'importance de la quantité de liquide mis en place pour assurer un développement normal des œufs.

Une seule insémination a été pratiquée durant l'œstrus pour une même Truie, les quelques truies qui ont été traitées 2 fois à $2 \mathrm{I}$ jours d'intervalle ont été notées comme deux truies distinctes, le résultat de la première insémination étant comptabilisé parmi les résultats négatifs.

Une visite faite à tous les propriétaires de truies inséminées 4 à 5 mois après l'intervention a permis de connaitre exactement les résultats.

Une centaine d'inséminations ont été réalisées d'autre part au Iaboratoire, sur des truies de Ioo $\mathrm{kg}$ parfaitement suivies. Les lots 
d'animaux utilisés dans ce cas, bien que faibles, étaient alors homogènes. De la comparaison entre ces derniers résultats, ceux que nous avons obtenus dans la pratique et ceux d'expériences réalisées dans des pays étrangers, nous nous efforcerons de dégager des conclusions qui sont susceptibles de guider des essais ultérieurs plus importants.

\section{2o Résultats}

\section{a) Le pourcentage de gestation.}

Parmi les 762 truies inséminées, I62 ont été éliminées comme notoirement inaptes à la reproduction (voir tableau III, pour r956). Sur les 600 autres, 207 ont mis bas, soit $34,5 \%$.

\section{TABLEAU III \\ (Truies éliminées, Yonne, I956)}

58 Truies éliminées dont :

7 traitées par injection de folliculine ;

24 saillies après insémination artificielle et non pleines;

27 divers :

- pleines avant insémination artificielle;

- saillies le lendemain de l'insémination artificielle;

- inséminées 2 fois dans le même oestrus ;

- notoirement déficientes ou malades;

-- pesant $60 \mathrm{~kg}$ ou moins.

Le nombre moyen de porcelets par portée fut de 9,0, valeur convenable, vu les conditions d'élevage des animaux, et identique à celle que rapporte MADDEN (1956) à la suite d'une expérience comportant un peu plus de 250 inséminations chez des éleveurs. Seulement II \% des portées furent composées de moins de 5 petits.

Tableau IV

\begin{tabular}{|c|c|c|c|c|c|}
\hline $\begin{array}{l}\text { Antécédents } \\
\text { des } \\
\text { Truies }\end{array}$ & $\begin{array}{l}\text { Nombre } \\
\text { de Truies } \\
\text { inséminées }\end{array}$ & $\begin{array}{c}\text { Nombre } \\
\text { de Truies ayant } \\
\text { mis-bas }\end{array}$ & $\begin{array}{l}\text { Taux } \\
\text { de gestation }\end{array}$ & $\begin{array}{c}\text { Nombre moyen } \\
\text { de porcelets } \\
\text { par portée }\end{array}$ & $\begin{array}{l}\text { Ecart } \\
\text { entre les } \\
\text { portées }\end{array}$ \\
\hline $\begin{array}{l}\text { Truies ayant déjà } \\
\text { porté............ } \\
\text { Truies n'ayant ja- } \\
\text { mais porté....... }\end{array}$ & $\begin{array}{l}326 \\
274\end{array}$ & $\begin{array}{r}I_{3} 8 \\
69\end{array}$ & $\begin{array}{l}42,3 \% \\
25,6 \%\end{array}$ & $\begin{array}{l}9,6 \\
7,7\end{array}$ & $\begin{array}{l}\mathrm{I}-\mathrm{I} 8 \\
\mathrm{I}-\mathrm{I} 4\end{array}$ \\
\hline Total... & 600 & 207 & $34,5 \%$ & 9,० & $\mathrm{I}-\mathrm{I} 8$ \\
\hline
\end{tabular}

Comme on le voit, le pourcentage de réussite est donc faible. Il est cependant sensiblement supérieur à celui qu'avaient obtenu CORONki en I953 (24\% sur 27 I truies), et, en I955 et I956, MADDEN ( $25 \%$ ), et HoL, T ( $4 \%$ ) en Angleterre. On ne peut le comparer aux résultats des Japonais qui utilisèrent toujours un sperme très peu dilué. Cependant, il reste très inférieur au chiffre de $63 \%$ que nous avons obtenu au labo- 
ratoire (30 truies) en utilisant $250 \mathrm{cc}$ de sperme dilué avec le dilueur bicarbonate-glucose en présence de jaune d'œuf ou de lait.

Certes l'hétérogénéité des animaux et la diversité des conditions d'insémination dans les fermes empêchent de rapprocher le chiffre global obtenu pour le pourcentage de gestation dans la pratique avec ce dernier résultat. Cependant parmi les inséminations faites chez les agriculteurs, 40 ont été réalisées dans des conditions comparables à celles que nous avons utilisées en laboratoire (jeunes truies de $100 \mathrm{~kg}$, plus de $6.10^{9}$ spermatozoïdes, conservation : moins de 3 heures) : $\mathbf{I}_{7}$ portées sont nées de ces 40 inséminations, soit un pourcentage de réussite de $42,5 \%$ qui reste donc encore sensiblement différent des $63 \%$ enregistrés en laboratoire.

La différence est due probablement à deux causes :

I $^{\circ}$ Les conditions peu favorables d'hygiène et d'alimentation où se trouvent placés souvent les animaux dans les fermes.

$2^{\circ}$ L'imperfection dans la détection des chaleurs. En effet les animaux utilisés en laboratoire furent présentés deux fois par jour à un Verrat boute-en-train : les chaleurs ont été donc définies exactement. Au contraire, dans les fermes, l'inséminateur est obligé de se fier aux dires de l'éleveur.

Cette différence entre les résultats à la ferme et ceux qu'on enregistre au laboratoire a été constatée par tous ceux qui ont abordé le problème dans les deux conditions. Ainsi PoLGE en Angleterre obtient $60 \%$ de réussite sur des truies bien connues alors qu'avec des méthodes comparables il n'obtint plus que $25 \%$ à $35 \%$ dans la pratique.

Ce dernier y voit comme nous-mêmes le manque d'habileté des petits éleveurs à repérer les chaleurs et les chiffres qu'il indique pour les pourcentages de mise-bas, en fonction de l'importance de la porcherie, sont particulièrement démonstratifs à cet égard (tableau V).

\section{TABLEAU V}

(D'après Polge et Rowson, I956).

Inportance
de la porcherie

Il est possible que cette amélioration soit due non seulement au fait que, si le propriétaire a beaucoup de truies il s'en occupe davantage et mieux, mais aussi au fait que probablement, parmi les éleveurs qui possèdent II truies et plus, beaucoup ont un verrat ce qui facilite la 
détection des chaleurs et place ce lot dans des conditions comparables à celles du laboratoire quant à la détection des chaleurs.

\section{b) Fréquence des suspensions de cycle œstrien}

Un deuxième fait qui semble liè à la pratique de l'insémination dans les fermes est la suspension des chaleurs chez certaines truies après insémination alors qu'elles ne sont pas pleines.

Comme le souligne POLGE, le pourcentage de non-retours à 3 mois, n'a aucun rapport avec le pourcentage de mise-bas et on relève dans ses résultats 60 à $65 \%$ de non-retours à 3 mois pour 25 à $35 \%$ de gestation effective. HoL $T$ ( I956) a enregistré $7 \mathrm{I} \%$ de non-retours à 3 semaines pour seulement I4 \% de mise-bas.

Pour notre part nous avons divisé les truies non gestantes en 2 catégories :

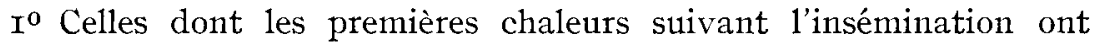
été repérées moins de 2 mois après l'intervention; le I $^{\text {er }}$ œstrus après 3 semaines n'a quelquefois pas été noté, mais en général les propriétaires de ces truies ne sont pas étonnés de cet état de chose. Nous avons considéré ces retours en chaleur comme "retours normaux ".

$2^{\circ}$ Les retours anormaux groupent tous les autres cas : les truies qui ont présenté des signes de chaleurs au bout de 3 mois ou au moment supposé du terme, aussi bien que celles qui ne sont jamais revenues en chaleurs après 4 mois et plus, et qui ont été abattues.

TABLEAU VI

Retours anormaux.

\begin{tabular}{c|c|c}
\hline $\begin{array}{c}\text { Nombre de Truies } \\
\text { inséminées }\end{array}$ & $\begin{array}{c}\text { Nombre de Truies } \\
\text { non gestantes }\end{array}$ & $\begin{array}{c}\text { Pourcentage de "retours anormaux " par rapport à l'en- } \\
\text { semble des " retours" }\end{array}$ \\
\hline 600 & 393 & $19,6 \%(77 / 393)$ \\
\hline
\end{tabular}

Ces stérilités très prolongées ou permanentes ont atteint environ I2 \% des truies inséminées (77 sur 600 inséminations) c'est-à-dire presque $20 \%$ des truies non-gestantes.

Tous les utilisateurs de l'insémination artificielle ne semblent pas avoir observé ces anomalies des chaleurs dans les mêmes proportions, puisque les auteurs japonais n'en parlent pas et que MADDEN signale seulement 2 cas pour 262 inséminations; 1'observation des chaleurs reste d'autre part liée à l'appréciation subjective de l'éleveur.

Il semble cependant que la notion même de cycle suspendu après l'insémination doit être retenue.

Or, au cours des essais faits sur des truies dont les chaleurs ont été testées par un Verrat, ce phénomène n'a jamais été constaté. 
En rapprochant la présence des " retours anormaux " et l'absence de Verrat boute-en-train pour tester les chaleurs, on pouvait supposer qu'il existât un rapport de cause à effet entre les inséminations faites à contre-cestrus et la suspension du cycle œstrien.

Afin d'infirmer ou de confirmer cette hypothèse, nous avons entrepris une série d'inséminations effectuées systématiquement à contretemps avec du sperme dilué comprenant comme au cours des inséminations pratiquées dans les fermes : 6 milliards de spermatozoïdes dans $25^{\circ}$ cc de diluat (3/4 bicarbonate-glucose, $I / 4$ jaune d'œuf, même dose d'antibiotique).

Dans ces conditions, même au moment où le corps jaune est pleinement fonctionnel, on n'obtient pas d'infection dans l'utérus, mais seulement un résidu plus ou moins sec, non purulent ( $\left.{ }^{1}\right)$.

Les cycles ultérieurs ne sont nullement suspendus, que l'insémination ait lieu avec ou sans antibiotique; et si nous avons constaté plusieurs fois des chaleurs plus courtes, toutes les truies saillies au cours de ces cycles ont mis bas ( 7 sur 7$)$.

En tout cas la question des retours anormaux reste entière. L'insémination faite à contre-œstrus ne suffit pas à expliquer la suspension de l'ostrus, quoique le contact journalier du Verrat et des Truies dont les chaleurs sont testées, peut provoquer une certaine stimulation des fonctions génitales, stimulation qui n'existe pas pour les truies élevées le plus souvent en l'absence de verrat, dans les fermes.

Ainsi la fréquence des retours anormaux dans la pratique jointe à la faiblesse des pourcentages de mise-bas en usant de sperme dilué, constitue un handicap sérieux pour 1'insémination artificielle porcine.

\section{IV. - LES FACTEURS DE VARIATION DU TAUX DE GESTATION}

\section{Dilueurs}

Le dilueur bicarbonate-glucose-jaune d'œuf qui nous avait donné de bons résultats au laboratoire, nous a servi de dilueur de référence. Sauf indication contraire, dans tous les dilueurs nous avons utilisé la dose maximum d'antibiotiques compatible avec la bonne conservation du sperme (DuMesnit, Du Buisson, I956). Nous avons à partir de ce dilueur (A) supprimé ou substitué un certain nombre des éléments.

$\mathrm{I}^{\mathrm{o}}$ Substitution de glycocolle à $3 \%$ à la solution saline, produisant un dilueur voisin de celui qu'a indiqué Roy (I955) et utilisé POLGE (I956) (B).

(1) Au contraire, les mêmes inséminations faites sans antibiotique ont provoqué des métrites très importantes, comme RowSON, LAMMING et FRY (IG5.3) le constatent chez la Vache inséminée à contreostrus et Mc Donald, Black, Mc NutT et Casida (I952) chez la Lapine, avec du sperme non aseptisé. 
TABLEAU VII

Composition des Dilueurs.

\begin{tabular}{|c|c|c|c|c|c|c|}
\hline \multirow[b]{3}{*}{ No } & \multirow{3}{*}{$\begin{array}{c}\text { Dilueurs } \\
\text { Dénomination }\end{array}$} & \multicolumn{5}{|c|}{ Proportion pour roo cc de dilueur } \\
\hline & & \multicolumn{2}{|c|}{ Solution aqueuse } & \multicolumn{2}{|c|}{ Milieu Biologique } & \multirow{2}{*}{$\frac{\begin{array}{c}\text { Antibio- } \\
\text { tique }\end{array}}{\text { Dose }}$} \\
\hline & & Désignation & Quantité & Désignation & Quantité & \\
\hline \multirow{9}{*}{$\begin{array}{l}A \\
B \\
C_{1} \\
C_{2} \\
D_{1} \\
D_{2} \\
E_{2} \\
F_{1}\end{array}$} & Bicarbonate-glucose Jo $N$. & II & & J. œuf & 25 & $\mathrm{Y}$ \\
\hline & Glycocolle Jo N. & 0 & 75 & J. ouf & 25 & $x$ \\
\hline & Bicarbonate-glucose, lait, $N$ & O & 75 & lait & 25 & $\mathrm{x}$ \\
\hline & Glycocolle, lait, N. & 0 & 75 & lait & 25 & $\mathrm{~N}$ \\
\hline & Bicarbonate-glucose, lait, $\mathrm{N} / 2$. & iI & 75 & lait & 25 & \\
\hline & Bicarbonate-glucose, Jo N/ $\mathrm{ro}$.. & M & 75 & J. œuf & 25 & $\mathrm{~N} / \mathrm{io}$ \\
\hline & $\begin{array}{l}\text { Bicarbonate-glucose I/20 Jo N. } \\
\text { Bicarbonate-glucose Jo N Der- }\end{array}$ & $\mathrm{M}$ & 95 & J. œuf & 5 & \\
\hline & $\begin{array}{c}\text { Bicarbonate-giucose Jo N. Der- } \\
\text { nier moment................... }\end{array}$ & $\mathrm{M}$ & 75 & J. œuf & 25 & $\mathrm{~N}$ \\
\hline & $\begin{array}{c}\text { Bicarbonate-glucose N. Dernier } \\
\text { moment ............................ }\end{array}$ & II & 100 & néant & & $x$ \\
\hline
\end{tabular}

Composition en gr your roo ce de solution aqueuse :

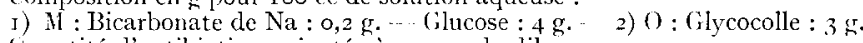

(Quantité d'antibiotique ajoutée à $100 \mathrm{cc}$ de dilueur :

$\hat{N}: 400 \mathrm{mg}$ de streptomycine et $240 \mathrm{mg}$ de pénicilline;

$\mathrm{N} / 2$ : Demi-dose ;

$\mathrm{N} / \mathrm{I} 0$ : Dixic̀me de la dose $\mathrm{N}$.

$2^{\circ}$ Substitution du lait écrémé chauffé pendant Io minutes à $90^{\circ}$ au jaune d'œuf, dans les deux premiers dilueurs $\left(C_{1}\right.$ et $\left.C_{2}\right)$.

$3^{\circ}$ Variation de la quantité d'antibiotique. Concentration ramenée à la demi-dose $\left(\mathrm{D}_{1}\right)$, au $\mathrm{I} /$ Io de la dose initiale $\left(\mathrm{D}_{2}\right)$.

$4^{0}$ Réduction de la quantité de jaune d'œuf qui passe de $25 \%$ à $5 \%$ du diluat $(\mathrm{E})$.

$5^{0}$ Variation du moment de la dilution, celle-ci étant opérée non plus tout de suite après la collecte, mais au moment de l'insémination, soit avec du dilueur normal $\left(F_{1}\right)$, soit avec le même, mais sans jaune d'œuf $\left(\mathrm{F}_{2}\right)$.

C'est dans les lots les plus réduits $\left(\mathrm{D}_{1}\right.$ et E) que le pourcentage de gestation s'écarte le plus de la moyenne, et aucune différence n'est significative (voir tableau VIII).

Entre les 4 lots les plus importants $\left(\mathrm{A}, \mathrm{B}, \mathrm{C}_{1}\right.$ et $\left.\mathrm{D}_{2}\right)$ on ne relève que des différences de réussite faibles, qui font penser que l'on peut employer les 4 dilueurs indifféremment.

Nous n'avons pratiqué aucune insémination sans antibiotique. Mais sur la nécessité des antibiotiques, les résultats de HoLt sont réritablement éloquents : $8,7 \%$ d'inséminations réussies ( 276 inséminations) sans antibiotique, contre $25,7 \%$ (89 inséminations) avec pénicilline et streptomycine.

La quantité d'antibiotiques employée dans le lot $\mathrm{D}_{2}$ semble assurer des résultats équivalents à la dose dix fois plus forte utilisée partout 
TABLEAU VIII

\begin{tabular}{|c|c|c|c|c|c|}
\hline & Dilueurs & & $\begin{array}{c}\text { Nombre } \\
\text { de truies } \\
\text { inséminées }\end{array}$ & $\begin{array}{l}\text { Nombre } \\
\text { de truies } \\
\text { pleines }\end{array}$ & $\begin{array}{l}\% \text { de truies } \\
\text { plcines }\end{array}$ \\
\hline \multirow{2}{*}{$\begin{array}{c}\text { Dilueurs à base de jaune } \\
\text { d'œuf } \\
\text { Dose normale } \\
\text { d'antibiotiques }\end{array}$} & $\begin{array}{l}\text { Bicarbonate Glucose } \\
\text { Jaune d'ouf }\end{array}$ & $A$ & 209 & 70 & $33,4 \%$ \\
\hline & Glycocolle Jaune d'œuf & $\mathrm{B}$ & 127 & 45 & $35,4 \%$ \\
\hline \multirow{2}{*}{$\begin{array}{l}\text { Dilueurs à base de lait } \\
\text { Dose normale } \\
\text { d'antibiotiques }\end{array}$} & Bicarbonate Glucose Lait & $\mathrm{C}_{\mathbf{I}}$ & 74 & 28 & $37,8 \%$ \\
\hline & Glycocolle Lait & $\mathrm{C}_{2}$ & $3^{\circ}$ & $\mathrm{I}_{4}$ & $46,6 \%$ \\
\hline \multirow{2}{*}{$\begin{array}{l}\text { Variation des doses } \\
\text { d'antibiotiques }\end{array}$} & $\begin{array}{c}\text { Bicarbonate Glucose Iait } \\
\mathrm{N} / 2\end{array}$ & Dr & 26 & 7 & $26,9 \%$ \\
\hline & $\begin{array}{l}\text { Bicarbonate Glucose } \\
\text { Jaune d'œuf N/IO }\end{array}$ & $\mathrm{D}_{2}$ & 47 & I 7 & $3^{6,1} \%$ \\
\hline $\begin{array}{l}\text { Variation du pourcentage } \\
\text { de jaune d'œuf }\end{array}$ & $\begin{array}{l}\text { Bicarbonate Glucose } \\
\text { I } 20 \text { de Jaune d'œuf }\end{array}$ & $\mathrm{E}$ & 26 & 5 & $19,2 \%$ \\
\hline \multirow{3}{*}{$\begin{array}{l}\text { Conservation du sperme pur } \\
\text { Dilution au moment } \\
\text { de l'insémination }\end{array}$} & $\begin{array}{l}\text { Bicarbonate Glucose } \\
\text { Jaune d'ouf }\end{array}$ & $F_{I}$ & $3^{\mathrm{I}}$ & 9 & $29,2 \%$ \\
\hline & Bicarbonate Glucose & $\mathrm{F}_{2}$ & 30 & I 2 & $40,2 \%$ \\
\hline & Total & & 600 & 207 & $34,2 \%$ \\
\hline
\end{tabular}

ailleurs. Elle représente par insémination Ioo ooo U. I. de pénicilline et o, I $\mathrm{g}$ de streptomycine, donc une dépense faible.

L'emploi de la poudre de lait serait également plus économique que celui du jaune d'œuf. Les résultats que nous rapportons ici sont tout à fait comparables $\left(A\right.$ et $\left.C_{1}\right)$ que l'on emploie l'un ou l'autre de ces deux milieux biologiques dans le dilueur. Nous recommanderions l'usage du lait si nous n'avions constaté, au cours d'essais effectués au laboratoire, de très grandes irrégularités dans le nombre de gestations avec la poudre de lait. Il serait probablement nécessaire d'employer un lait de grand mélange.

La comparaison entre les résultats dans le lot B (glycocolle jaune d'œuf) et dans le lot A ne fait pas ressortir de différences significatives. Nous n'avons pas l'expérience des dilueurs citrates que HoL'T (I956) a employés pour les 'Truies.

Ainsi le bicarbonate-glucose qui permet en mélange avec le lait ou le jaune d'œuf, une bonne conservation, et qui a servi ici pour 443 inséminations, peut donc être utilisé avec une certaine sécurité dans la pratique de l'insémination porcine.

Enfin, le pourcentage de gestation obtenu dans le lot de truies traitées avec du sperme dilué au moment de l'insémination, est le même que dans le lot avec le dilueur de référence. Quoique le nombre des animaux y soit plus faible, ce résultat semble indiquer que le pouvoir fécondant est conservé au moins aussi bien dans le sperme pur que dans le sperme 
dilué. Ceci ne fait, du reste, que confirmer la conclusion que l'on peut tirer des résultats des Japonais ITo, Nrwa et Kudo (r948 b).

\section{$2^{\circ}$ Durée de conservation du sperme}

La chute que l'on enregistre (voir tableau IX, trois dernières colonnes) dans le pourcentage de gestation sur l'ensemble des animaux montre que même dans les limites que nous nous sommes imposées pour la durée de conservation du sperme, cette dernière intervient de façon très nette : de $39 \%$ à moins de 3 heures, on passe à $29 \%$ à plus de 6 heures.

Tableau IX

\begin{tabular}{|c|c|c|c|c|c|c|c|c|c|}
\hline \multirow{2}{*}{$\begin{array}{c}\text { Durée } \\
\text { de conservation }\end{array}$} & \multicolumn{3}{|c|}{ Truies ayant déjà porté } & \multicolumn{3}{|c|}{$\begin{array}{c}\text { Truies n'ayant jamais } \\
\text { porté }\end{array}$} & \multicolumn{3}{|c|}{ Total des truies inséminées } \\
\hline & $\begin{array}{l}\text { Truies in- } \\
\text { séminées }\end{array}$ & $\begin{array}{l}\text { Truies } \\
\text { pleines }\end{array}$ & $\begin{array}{c}\% \text { de } \\
\text { truies } \\
\text { pleines }\end{array}$ & $\begin{array}{l}\text { Truies in- } \\
\text { séminées }\end{array}$ & $\begin{array}{l}\text { Truies } \\
\text { pleines }\end{array}$ & $\begin{array}{c}\% \text { de } \\
\text { truies } \\
\text { pleines }\end{array}$ & $\begin{array}{l}\text { Truies in- } \\
\text { séminées }\end{array}$ & $\begin{array}{l}\text { Truies } \\
\text { pleines }\end{array}$ & $\begin{array}{l}\% \text { de } \\
\text { truies } \\
\text { pleines }\end{array}$ \\
\hline $\begin{array}{l}\text { Moins de } 3 \text { h.... } \\
\text { De } 3 \text { h à } 6 \text { h. . } \\
\text { Plus de } 6 \text { h.... }\end{array}$ & $\begin{array}{r}108 \\
163 \\
55\end{array}$ & $\begin{array}{l}48 \\
70 \\
20\end{array}$ & $\begin{array}{l}44,4 \% \\
42,9 \% \\
36,4 \%\end{array}$ & $\begin{array}{r}89 \\
127 \\
58\end{array}$ & $\begin{array}{l}30 \\
26 \\
13\end{array}$ & $\begin{array}{l}33,7 \% \\
20,5 \% \\
22,4 \%\end{array}$ & $\begin{array}{l}197 \\
290 \\
\text { II } 3\end{array}$ & $\begin{array}{l}78 \\
96 \\
33\end{array}$ & $\begin{array}{l}39,5 \% \\
33,1 \% \\
29,2 \%\end{array}$ \\
\hline Total ... & 326 & 138 & $42,3 \%$ & 274 & 69 & $25,6 \%$ & 600 & 207 & $34,5 \%$ \\
\hline
\end{tabular}

Une chute spectaculaire avait déjà été observée par PoLGE au bout de $I$ et 2 jours, les pourcentages de fécondation, dans les conditions du laboratoire où il opérait, tombant de $60 \%$ à $35 \%$ à 24 heures et à Io $\%$ après 48 heures (sur 28 truies).

Dans ce dernier cas comme pour la majorité des truies inséminées par nous-mêmes, le sperme avait été conservé dilué dans un milieu à base de jaune d'œuf assurant un bon maintien de la survie et de la motilité. En inséminant avec du sperme conservé pur, ITo, NIWA et Kudo, bien que leurs résultats soient meilleurs, ont enregistré également une chute : 69, I $\%$ à moins de 24 heures, $55,3 \%$ entre 24 et 48 heures, et $34,7 \%$ entre 48 et 75 heures.

Nos résultats soulignent la rapidité avec laquelle intervient l'abaissement du pouvoir fécondant, aussi devons-nous conclure qu'en inséminant systématiquement dans les 3 heures qui suivent la collecte on peut augmenter de façon sensible le pourcentage de réussite.

\section{$3^{\circ}$ Nombre de spermatozoïdes par insémination}

Nous avons groupé les truies suivant le nombre de spermatozoïdes qui ont été utilisés par insémination. De l'examen des résultats globaux (voir les 3 dernières colonnes du tableau $\mathrm{X}$ ), on peut conclure immédiatement à l'influence de ce nombre sur le pourcentage de gestation, bien que ce nombre ait été dans tous les cas supérieur à 2 milliards. 
Il n'en est pas du tout de même lorsqu'il s'agit seulement d'obtenir la fécondation des œufs et les chiffres minima qu'indiquent WIGG INS Grumimer et CASIDA (I95I) pour obtenir une fécondation sont tout à fait d'un autre ordre : avec 0,2 milliard de spermatozoïdes, plus de $70 \%$ des truies ont des oufs fécondés.

Pour avoir des taux de gestation comparables, il faut au moins 20 fois plus de spermatozoïdes : ainsi sur 9 inséminations faites avec moins de 2 milliards de spermatozoïdes, POLGE n'a obtenu qu'une gestation et ITo, Niwa et KUDo voient leur pourcentage de réussite tomber de $76,8 \%$ à $55 \%$ quand le nombre de spermatozoïdes employé est inférieur à 4 milliards.

\section{TABLEAU X}

\begin{tabular}{|c|c|c|c|c|c|c|c|c|c|}
\hline \multirow{2}{*}{$\begin{array}{c}\text { Nombre de } \\
\text { spermatozoides } \\
\text { par insémination }\end{array}$} & \multicolumn{3}{|c|}{ Truies ayant déjà porté } & \multicolumn{3}{|c|}{$\begin{array}{c}\text { Truies n'ayant jamais } \\
\text { portê }\end{array}$} & \multicolumn{3}{|c|}{ Total des truies inséminées } \\
\hline & $\begin{array}{l}\text { Truies in- } \\
\text { séminées }\end{array}$ & $\begin{array}{l}\text { Truies } \\
\text { pleines }\end{array}$ & $\begin{array}{l}\% \text { de } \\
\text { truies } \\
\text { pleincs }\end{array}$ & $\begin{array}{l}\text { Truies in- } \\
\text { séminéts }\end{array}$ & $\begin{array}{l}\text { Truies } \\
\text { pleines }\end{array}$ & $\begin{array}{c}\% \text { de } \\
\text { truies } \\
\text { pleines }\end{array}$ & $\begin{array}{l}\text { Truies in- } \\
\text { séminćes }\end{array}$ & $\begin{array}{l}\text { Truies } \\
\text { pleines }\end{array}$ & $\begin{array}{l}\% \text { de } \\
\text { truies } \\
\text { pleines }\end{array}$ \\
\hline $\begin{array}{l}2 \text { à } 4 \cdot 10^{9} \ldots . \\
4 \text { à } 5,9 \cdot 10^{9} . \\
6 \text { à } 9,9 \cdot 10^{9} . \\
\text { ro à } 14 \cdot 10^{9} .\end{array}$ & $\begin{array}{r}105 \\
9 \mathrm{I} \\
80 \\
50\end{array}$ & $\begin{array}{l}37 \\
39 \\
36 \\
\mathbf{2 6}\end{array}$ & $\begin{array}{l}35,2 \% \\
42,8 \% \\
45,0 \% \\
52,0 \%\end{array}$ & $\begin{array}{l}77 \\
82 \\
69 \\
46\end{array}$ & $\begin{array}{l}\text { II } \\
2 \mathrm{I} \\
24 \\
\text { I3 }\end{array}$ & & $\begin{array}{r}\mathbf{1} 82 \\
173 \\
\mathbf{1} 49 \\
96\end{array}$ & $\begin{array}{l}48 \\
6 \circ \\
60 \\
39\end{array}$ & \\
\hline otaus & 326 & $3^{8}$ & $3 \%$ & 274 & 69 & $25,1 \%$ & 600 & 207 & $34,5 \%$ \\
\hline
\end{tabular}

Cependant il est possible qu'en modifiant le mode d'insémination on puisse réduire beaticoup le nombre de spermatozoïdes à introduire. En effet, une expérience d'insémination directe au sommet des cornes utérines après laparotomie nous a montré que la gestation pouvait elle aussi être réalisée avec des nombres de spermatozoïdes inférieurs à 0,2 milliard.

On peut donc penser qu'il conviendrait par un artifice d'amener rapidement au voisinage de la jonction utéro-tubaire, la plupart des spermatozoïdes inséminés, peut-être en fractionnant, comme au cours de 1'éjaculation, 1'inséminat. Il est possible que, lors de l'accouplement, le " tapioca " joue un rôle dans le maintien à l'intérieur de l'utérus, du fractionnement qui se produit pendant l'éjaculation.

Il semble également que la diminution du nombre de spermatozoïdes inséminés entraîne une augmentation de la fréquence des portées comprenant seulement I ou 2 porcelets.

\section{$4^{\circ}$ Verrats et antécédents de reproduction des Truies}

Il existe certainement des différences de fertilité entre Verrats mais le nombre de Truies inséminées est trop faible pour les mettre en évidence. 
Pour les truies, la différence globale de pourcentage qui apparait entre les truies qui n'ont jamais porté (274) et les autres (326) est très importante et tout à fait significative : $25,6 \%$ contre $42,3 \%$ (tableau IV).

Les résultats publiés par PoLGE en I956, d'essais en laboratoire, font ressortir également un taux de gestation plus élevé sur les truies qui ont déjà porté $(79 \%$ sur I9 inséminations contre $50 \%$ sur 46 inséminations). Au contraire, Hor $T$ dans la pratique de l'insémination, ne trouve aucune différence entre les jeunes truies et les vieilles. Dans les conditions où nous opérons, les causes d'infécondité seraient donc plus nombreuses chez les truies de Ioo $\mathrm{kg}$.

Comme le montrent les tableaux IX et $\mathrm{X}$, ce sont les jeunes truies qui sont les principales responsables de 1'affaiblissement du pourcentage de réussite, en fonction de la durée de conservation et du nombre de spermatozoïdes : $21 \%$ (I 85 inséminations) contre 33,7\% (89 inséminations) quand on prolonge la conservation du sperme au-delà de 3 heures ; $14,2 \%$ (77 inséminations) contre $29,4 \%$ (I97 inséminations) quand on abaisse le nombre de spermatozoïdes en dessous de 4 milliards. Pour les truies qui ont déjà porté, la chute est plus faible.

Comme après accouplement naturel, on constate que le nombre moyen de porcelets par portée est plus important chez les multipares $(9,6)$ que chez les primipares $(7,7)$; ceci est dû en partie à la fréquence plus grande des portées de $I_{5}$ et plus chez les multipares.

Aussi pour la pratique de l'insémination, dans des conditions de dilution et d'insémination comparables à celles où nous nous sommes placés dans tous ces essais, il sera utile d'employer systématiquement un nombre de spermatozoïdes compris entre 4 et ro milliards et d'inséminer rapidement - si possible dans les trois heures - après la collecte.

\section{CONCL USION}

Après avoir obtenu des taux de gestation très convenables en laboratoire par insémination artificielle des truies, il était utile de connaître quels résultats on pouvait escompter de la transposition des mêmes techniques dans la pratique.

Un certain nombre de problèmes nouveaux se posent alors : entre autres celui de la détection des chaleurs et de la suspension du cycle œstrien après insémination infructueuse.

Tout en faisant ressortir un taux de gestation moyen assez faible, cette expérience a mis en lumière l'existence de deux des facteurs principalement responsables de l'abaissement de ce taux de gestation : durée de conservation et nombre de spermatozoïdes inséminés.

Ën se plaçant dans les conditions suivantes : durée de conservation ne dépassant pas trois heures, insémination avec au moins $4 \times \mathrm{IO}^{9}$ 
spermatozoïdes, on peut escompter une amélioration sensible des résultats pratiques, en conservant les techniques actuelles.

Le maintien du pouvoir fécondant du sperme en fonction des conditions de conservation ne pourra être étudié que par l'insémination d'un très grand nombre de truies. On peut dire qu'actuellement rien n'autorise à penser que le sperme dilué avec les dilueurs biologiques classiques conserve mieux son pouvoir fécondant que le sperme pur.

Le nombre relativement élevé de spermatozoïdes que l'on doit introduire à l'heure actuelle pourra probablement être abaissé par un fractionnement de l'inséminat au moment de l'insémination.

Ainsi ces résultats assignent actuellement à l'insémination artificielle porcine une limite quant au nombre d'inséminations que l'on peut espérer à partir d'un Verrat; on peut parler raisonnablement de 500 portées par verrat et par an, chiffre déjà très supérieur à la moyenne d'utilisation des verrats en France ( $I$ verrat pour 20 truies), mais on se trouve encore fort loin des 20000 inséminations avancées par PoLGE.

Le fonctionnement expérimental d'un Centre, pendant un an ou plus, doit nous apporter les éléments économiques qui nous manquent pour juger des possibilités d'extension de cette technique.

\section{Reçu pour publication le I6 avril I957.}

\section{RÉFÉRENCES BIBLIOGRAPHIQUES}

Aamdal (J.). - Cité par Madden (D. H. L.), Vet. Rec., 68, 959, I956.

Burger (J. F.). - Onderstepoort J. Vet. Res., suppl. no 2, I952.

Castor. - Soc. Fr. Gynecol. (mai-sept., no 5,) I950.

Corner (G. W.). - Carnegie Inst. Contribut. to Embryol., 2, 69-94, 1915.

CORNER (G. W.). - Carnegie Inst. Contribut. to Embryol., 13, II7-I46, I92I.

Coronel (A. B.). - Philipp. J. Anim. Ind., 14, 227-234, ABA 23, 65, I953.

Du Mesnil du Buisson (F.). - Ann. Zootech., série D, 3, I95-2I2, I 956.

Du Mesnil du Buisson (F.). - Ann. Zootech., série D, 4, 39I-399, I957.

Du Messil du Buisson (F.), Dauzier (L.). - C. R. Soc. Biol., 149, 76-79, I955 $a$.

Du Mesnil du Buisson (F.), Dauzier (L.). - Ann. End., 16, 4I3-422, I955 $b$.

Du Mesnil, du Buisson (F.), Dauzier (L.). - C. R. Acad. Sci., 241, I867I $869, \mathrm{I} 955 c$.

Holt (A. F.). - Vet. Rec., 68, 960, I956.

Ito (S.), Niwa ('T.) et Kudo (A.) - Zootech. exp. Sta. Res. Bull., 55, I-I5, I948 a.

Ito (S.), Niwa (T.) et Kudo (A.). - Zootech. exp.Sta. Res. Bull., 55, 57-74, I948 b.

Ito (S.), Niwa (T.), Kudo (A.) et Mrzuho (A.). - Zootech. exp. Sta. Res. Bull., 55, I6-56, I 948 .

Kvasniski (A. V.). - Rev. Physiol., URSS, 244-247, I95I.

L.EBedev (M. M.) et PitkJAnen (I. G.). - Sovetsk. Zootech., 6, 34-43, ABA 20, 57, x95I.

Madden (D. H. L.). - Vet. Rec., 68, 958-960, I956.

MANN (T.). - Nature, 168, I043-I044, I95I.

Mann (T.), Polge (C.) et Rowson (L. E.). - J. Endocrinol., 13, I33-I40, I956. 
Maureon (P.). - Bull. Interieur du Herd Book du Porc Large-White, Paris, 14, 48I-487, I953.

Mc Donald (L. E.), Black (W. G.), Mc Nutt (S. H.) et Casida (L. E.). Am. J. Vet. Res., 13, 4I9-424, I952.

Mc Kenzie (F. F.). - J. Am. Vet. Med. Ass., 78, 244-246, I93I.

Mc Kenzie (F. F.), Mriler (J. C.) et Bauguess (L. C.). - Mo Agric. Exp. Sta. Bull., 299, I938.

Milovanov (V. K.). - Prob. Zhivotn., 4, 3I-34, I932.

Milovanov (V. K.). - Art. insemination in Livestock, Moscou, A. B. A., 2, 403, I934.

Milovanov (V. K.) et Habibulin (K. H.). - Prob. Zhivotn., 5, 3I-34, A. B. A., $1, \mathrm{n}^{0} 4$, I933.

Polge (C.). - Vet. Rec., 68, 62-76, I956.

POLGE (C.) et Rowson (L. E.). - Vet. Rec., 68, 952-956, I956.

RODOLFO (A.). - Phillip. J. Sci., 53, I83-203, I934a.

Rodolfo (A.). - Phillip. J. Sci., 55, I3-18, I934 b.

Rowson (L. E.), Lamming (G. E.) et FRy (R. M.). - Vet. Rec., 65, 335, I953.

Roy (A.). - Vet. Rec., 67, 330-33I, I955.

Steinbach (G.). - Diss. Munich. A. B. A., 21, I70, I95I.

Thiery (G.). - Prix Emile Thiery Ac. Veter. Paris, I952.

Wiggins (E. L.), Grummer (R. H.) et Casida (L. E.). - J. An. Sci., 10, I $38-I 43$, I95I.

Wilson (K. M.). - Am.J.Anat., 3\%, 4I7-432, I926.

\author{
I.N.R.A. \\ BIBLIOTHEQUE UO 35906 \\ Domaine oE CROUELLE \\ 63039 \\ Clermant-FD CEDEX $?$
}

